\title{
Poisson Processes on Groups and Feynman Path Integrals
}

\author{
Ph. Combe ${ }^{1}$, R. Høegh-Krohn ${ }^{4}$, R. Rodriguez ${ }^{1}$, M. Sirugue ${ }^{2}$, \\ and M. Sirugue-Collin ${ }^{3}$ \\ 1 Faculté des Sciences de Luminy and Centre de Physique Théorique \\ 2 Centre de Physique Théorique \\ 3 Université de Provence and Centre de Physique Théorique, CNRS, Marseille, France, and \\ 4 Matematisk Institutt, Universitet i Oslo, Oslo
}

\begin{abstract}
We give an expression for the perturbed evolution of a free evolution by gentle, possibly velocity dependent, potential, in terms of the expectation with respect to a Poisson process on a group. Various applications are given in particular to usual quantum mechanics but also to Fermi and spin systems.
\end{abstract}

\section{Introduction}

Since the proposal of Feynman [1] of a quantization procedure by means of path integrals, a great deal of effort has been devoted to make precise the definition of such an integral (see e.g., $[2,3]$ for references). An important step has been to realize that in some cases this path integral can be interpreted as the expectation with respect to a stochastic process [4]. One of the most natural stochastic processes which has been considered first is the Gaussian process. Another one is the Poisson process $[5,6]$. In this paper, we shall consider the connection between Poisson processes on groups and the usual Weyl quantization procedure. More specifically, let $H_{0}$ be the free hamiltonian of a single particle and $V$ a multiplicative potential which is the Fourier transform of a bounded measure $v$. In the " $p$ " representation $H_{0}$ is just a multiplication operator by $h_{0}(p)=p^{2}$, then for any square integrable, continuous function $\psi$ one has

$$
\left\{e^{-i T\left(H_{0}+V\right)} \psi\right\}(p)=E_{p(T)=0}^{T}\left\{e^{-i \int_{0}^{T} h_{0}(p-p(\tau)) d \tau} \psi(p-p(0))\right\}
$$

where $E_{p(T)=0}^{T}$ denotes the expectation value with respect to a Poisson process naturally associated to $v$, Proposition (3.7).

In fact this result is a special case of a more general result. Let $G$ be a topological group which acts on a topological space $\mathfrak{X}$, and let $\mu$ be a quasiinvariant measure on $\mathfrak{X}$ with respect to the action of $G$. Under these assumptions the action of $G$ on $\mathfrak{X}$ induces $*$-automorphisms of $L_{\infty}(\mathfrak{X}, \mu)$ which are unitarily implemented by a unitary projective representation $U$ of $G$. Let $\mathfrak{M}$ be the cross product of $L_{\infty}(\mathfrak{X}, \mu)$ by the action of $G$. Then it is possible to write a formula of the 
previous type for the one parameter group whose generators are of the form $H_{0}+V$ where $H_{0}$ is an operator affiliated to $L_{\infty}(\mathfrak{X}, \mu)$ and $V$ is of the form $\int_{G} d v(g) U(g)$ with $v$ a bounded measure on $G$ (Theorem 2.11).

The first section is devoted to the unitary projective representations of a topological group $G$ induced by its action on a topological space $\mathfrak{X}$. In particular it is possible to give a fairly complete description of these representations when $G$ is abelian, acts transitively on $\mathfrak{X}$, and $\mathfrak{X}$ can be identified to a subgroup of $G$ (Proposition 1.9).

In Sect. 2, we give a definition of Poisson processes on a group $G$ associated to bounded measures $v$ on $G$. In particular we describe the sample paths space, which is the set of left continuous, piecewise constant, with a finite number of jumps, functions from $t \in[0, T]$ to $G$, ending at the origin. This leads to main theorem (2.11). More specific potentials with definite support properties are investigated.

The last two sections are concerned with specific groups $G$, namely abelian groups of the form $\mathfrak{G}_{0} \times \mathfrak{G}_{1}$ where $\mathfrak{G}_{1}$ is a subgroup of $\hat{\mathfrak{G}}_{0}$ the dual of $\mathfrak{G}_{0}$.

In the third section, $\mathfrak{G}_{1}=\hat{\mathfrak{G}}_{0}$ with the two examples:

i) $\mathfrak{G}_{0}=\mathbb{R}_{N}$ with the usual topology. This is nothing but ordinary quantum mechanics with $N$ degrees of freedom. The main theorem allows to treat some velocity dependent potentials namely those of the form

$$
V=\int_{\mathbb{R}_{2 N}} d v(x, p) U(x, p)
$$

where $U(x, p)$ are the ordinary Weyl-operators $e^{i(x P-p Q)}$ and $v$ a bounded measure on $\mathbb{R}_{2 N}$ then

$$
\begin{aligned}
\left(e^{-i T\left(H_{0}+V\right)} \psi\right)(p)= & E_{x(T)=p(T)=0}^{T} \\
& \cdot\left\{e^{-i \int_{0}^{T} h_{0}(p-p(\tau)) d \tau} \cdot e^{-i \int_{0}^{T} p(\tau) d x(\tau)} \cdot e^{-i p x(0)} \psi(p-p(0))\right\} .
\end{aligned}
$$

ii) $\mathfrak{F}_{0}=\mathfrak{P}(\Lambda)$ the group of finite subsets of a countable set with symmetric difference as group law. That gives models for spin systems in the usual Fock representation.

Fourth section deals with "quantum field like models" where $\mathfrak{G}_{1} \subsetneq \hat{\mathfrak{G}}_{0}$. The specific examples which are given are the following:

i) $\mathfrak{G}_{0}=\mathbb{R}_{N}$ with the discrete topology, $\mathfrak{G}_{1}=\mathbb{R}_{N}$.

Beside ordinary representations of $G$ there exist representations in non separable Hilbert spaces which simulate representations with position or momentum eigenvectors.

ii) $\mathfrak{G}_{0}=\mathfrak{P}(\Lambda)$ as previously with $\Lambda$ strictly infinite and $\mathfrak{b}_{1}=\mathfrak{G}_{0}$. It is possible to describe in this context the Fermi anti-commutation relations. In particular, we apply the main theorem to bounded perturbations of the number operator.

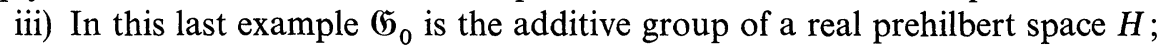
$\mathfrak{G}_{1}=\mathfrak{G}_{0}$. The main theorem applies but the fact that the potential has to be the Fourier transform of a bounded measure introduces strong restrictions.

There are many interesting applications of the previous general theory for more specific models which will be treated in separate forthcoming papers. 


\section{Unitary Projective Representations Corresponding to Group Action}

Let $G$ be a topological group and $\mathfrak{X}$ be a topological space. A continuous action of $G$ on $\mathfrak{X}$ is a continuous mapping (denoted multiplicatively) from $\mathfrak{X} \times G$ to $\mathfrak{X}$ which satisfies

$$
x \cdot e=x, \quad \forall x \in \mathfrak{X},
$$

where $e$ is the identity of $G$

$$
\left(x g_{1}\right) \cdot g_{2}=x \cdot\left(g_{1} g_{2}\right), \quad \forall g_{1}, g_{2} \in G, \quad \forall x \in \mathfrak{X} .
$$

Let $\zeta$ be a multiplier on $G$, i.e. a continuous function from $G \times G$ in the torus $\mathbb{T}$ which satisfies

$$
\zeta\left(g_{1}, g_{2}\right) \zeta\left(g_{1} g_{2}, g_{3}\right)=\zeta\left(g_{2}, g_{3}\right) \zeta\left(g_{1}, g_{2} g_{3}\right) \text {. }
$$

Let $\mu$ be a positive measure on $\mathfrak{X}$ which is quasi-invariant for the action of $G$ on $\mathfrak{X}$. Then it is possible to build a unitary projective representation of $G$ according to the following proposition (see e.g. [7]).

Proposition 1.4. Any continuous function $Z$ from $\mathfrak{X} \times G$ into $\mathbb{T}$ which satisfies

$$
Z\left(x, g_{1}\right) Z\left(x g_{1}, g_{2}\right)=\zeta\left(g_{1}, g_{2}\right) Z\left(x, g_{1} g_{2}\right)
$$

defines a continuous unitary projective representation $U^{Z}$ of $G$ on $L_{2}(\mathfrak{X}, d \mu)$ :

b)

$$
\left(U^{Z}(g) f\right)(x)=Z(x, g) \sqrt{\frac{d \mu(x g)}{d \mu(x)}} f(x g) .
$$

Conversely if $U^{Z}$ is a unitary projective representation of $G$ on $L_{2}(\mathfrak{X}, d \mu)$ defined by $b$, $Z$ satisfies property a).

$G$ has a continuous transitive action on itself by right multiplication. To that action corresponds the regular unitary projective representation $U^{\zeta}$ defined as

$$
\left(U^{\zeta}(g) f\right)\left(g^{\prime}\right)=\zeta\left(g, g^{\prime}\right) \sqrt{\frac{d \mu\left(g^{\prime} g\right)}{d \mu\left(g^{\prime}\right)}} f\left(g^{\prime} g\right)
$$

for a quasi (right) invariant measure $\mu$ on $G$.

This representation is unique in the following sense.

Proposition 1.6. Let $G$ be a topological group and $\mu$ a positive (right) quasiinvariant measure on $G$. Let $U^{Z}$ be a projective unitary representation of $G$ on $L_{2}(G, d \mu)$ corresponding to the function $Z$ defined in Proposition 1.4. Then $U^{Z}$ is unitarily equivalent to $U^{\zeta}$ and the intertwining operator $V$ between $U^{Z}$ and $U^{\zeta}$ is of the following form

$$
(V f)(g)=v(g) f(g), \quad f \in L_{2}(G, d \mu)
$$

for $v$ a continuous function from $G$ to $\mathbb{T}$.

The proof uses the following lemma.

Lemma 1.7. Let $Z$ be a (continuous) function from $G \times G$ to the torus $\mathbb{T}$ which satisfies

$$
Z\left(g_{1}, g_{2}\right) Z\left(g_{1} g_{2}, g_{3}\right)=\zeta\left(g_{2}, g_{3}\right) Z\left(g_{1}, g_{2} g_{3}\right)
$$


Then there exists a (continuous) function $v$ from $G$ to $\mathbb{T}$ such that

$$
Z\left(g, g^{\prime}\right)=\zeta\left(g, g^{\prime}\right) v(g) \overline{v\left(g g^{\prime}\right)}
$$

Let now $G$ be an abelian group which acts transitively on $\mathfrak{X}$, and $\mu$ a positive quasi-invariant measure on $\mathfrak{X}$. The existence of a unitary projective representation implies some restrictions on the multiplier $\zeta$.

Proposition 1.8. Let $G$ be an abelian topological group which acts transitively on $a$ topological space $\mathfrak{X}$, and $G_{0}$ be the stabilizer of the points in $\mathfrak{X}$. Let $\zeta$ be a multiplier on $G$. Assume there exists $Z$ satisfying property Proposition $1.4 \mathrm{a}$ ), then $\zeta$ restricted to $G_{0} \times G$ is trivial in the sense that there exists a function $\lambda$ from $G$ to $\mathbb{T}$ such that

$$
\zeta\left(g_{0}, g\right)=\lambda\left(g_{0}\right) \lambda(g) \overline{\lambda\left(g_{0} g\right)}, \quad \forall g_{0} \in G_{0}, \quad g \in G .
$$

As a corollary the $U^{Z}\left(g_{0}\right), g_{0} \in G_{0}$, commute.

Conversely we can describe the unitary projective representations of $G$ corresponding to a closed subgroup $G_{0}$ on which $\zeta$ is trivial in the above mentioned sense, more precisely:

Proposition 1.9. Let $G$ be a topological abelian group such that $G=G_{0} \times G_{1}$; moreover let $\zeta$ be a multiplier on $G$ such that

$$
\begin{gathered}
\zeta\left(\left(g_{0}, e\right)\left(g_{0}^{\prime}, g_{1}^{\prime}\right)\right)=\lambda\left(g_{0}, e\right) \lambda\left(g_{0}^{\prime}, g_{1}^{\prime}\right) \overline{\lambda\left(g_{0} g_{0}^{\prime}, g_{1}^{\prime}\right)} \\
\forall g_{0}, g_{0}^{\prime} \in G_{0}, \quad \forall g_{1}^{\prime} \in G_{1},
\end{gathered}
$$

where $\lambda$ is a function from $G$ to the torus $\mathbb{T}$.

Consider the following action of $G$ on $G_{1}$

$$
k \cdot\left(g_{0}, g_{1}\right)=k g_{1}, \quad \forall k \in G_{1} .
$$

Let $\mu$ be a positive quasi-right invariant measure on $G_{1}$. Let $Z$ be a function from $G_{1} \times G$ in the torus $\mathbb{T}$ which defines a unitary projective representation of $G$ on $L_{2}\left(G_{1}, d \mu\right)$. Then $Z$ has the following structure

$$
\begin{gathered}
Z\left(k,\left(g_{0}, g_{1}\right)\right)=\overline{\lambda\left(e, g_{1}\right)} \lambda\left(g_{0}, g_{1}\right) b^{\zeta}\left(\left(g_{0}, e\right)\left(e, k^{-1}\right)\right) \\
\cdot \chi\left(g_{0}\right) \zeta\left((e, k)\left(e, g_{1}\right)\right) v(k) \overline{v\left(k g_{1}\right)},
\end{gathered}
$$

where $b^{\zeta}$ is the bicharacter defined by

$$
b^{\zeta}\left(g, g^{\prime}\right)=\zeta\left(g, g^{\prime}\right) \overline{\zeta\left(g^{\prime}, g\right)}
$$

$\chi$ a continuous character on $G_{0}$ and $v$ a continuous function on $G_{1}$ with values in $\mathbb{T}$.

Conversely, given $\chi$ a continuous character of $G_{0}$ and $v$ a continuous function from $G_{1}$ to $\mathbb{T}$, then the previous formula defines a function $Z$ which verifies Proposition 1.4a).

This proposition is an easy consequence of a more general theorem whose proof is given in the Appendix.

These representations are obvious generalizations of " $x$ " or " $p$ " representations of the usual quantum mechanics (see later). Nevertheless they are not necessarily irreducible, at least if $G_{0}$ is not $\zeta$ maximal abelian. 
In what follows we shall be interested in some algebras of bounded operators associated with these representations, the most natural being the von Neumann algebra $\Delta^{U^{Z}}(G, \zeta)$ generated by the $U^{Z}(g)$. Furthermore, the $U^{Z}(g)$ implement the group of automorphisms of $L_{\infty}(\mathfrak{X}, d \mu)$ defined by

$$
\left(\theta_{g} M\right)(x)=M(x g) \quad \mu \text {-a.e. }
$$

that is

$$
\theta_{g} M=U^{Z}(g) M U^{Z}(g)^{*}
$$

[the same notation is used for an element of $L_{\infty}(\mathfrak{X}, d \mu)$ and the associated operator on $\left.L_{2}(\mathfrak{X}, d \mu)\right]$.

So, another interesting algebra is the von Neumann algebra $\mathfrak{M}$ generated by both the $U^{Z}(g)$ and the elements of $L_{\infty}(\mathfrak{X}, d \mu)$, i.e. the cross product of $L_{\infty}(\mathfrak{X}, d \mu)$ by the action (1.10) of $G$. Let us remark that in some cases $\mathfrak{M}$ is identical to $\Delta^{U^{Z}}(G, \zeta)$.

Now, we define a dense subalgebra $Q$ of $\Delta^{U^{z}}(G, \zeta)$ in the following way.

Definition 1.11. $Q$ is the *-algebra of elements in $\Delta^{U^{z}}(G, \zeta)$ of the form

$$
Q(v)=\int_{G} d v(g) U^{Z}(g),
$$

where $v$ is a bounded measure on $G$.

This is a generalization of the Weyl quantization procedure. In this case $G$ is an abelian locally compact group, $\hat{G}$ its dual and $d g$, $d \hat{g}$ the corresponding Haar measures. Let $\zeta$ be a multiplier on $G$, the associated bicharacter $b^{\zeta}$ on $G$ ensures a homomorphism of $G$ into its dual $\hat{G}$. Let us assume, that is the case of ordinary quantum mechanics, that this morphism is an isomorphism, then

$$
(\mathscr{F} f)(g)=\int_{\hat{G}} b^{\zeta}(g, \hat{g}) f(\hat{g}) d \hat{g}
$$

defines a Fourier transform of functions on $\hat{G}$. The operator $Q^{\omega}(f)$ defined by

$$
Q^{\omega}(f)=\int_{G}(\mathscr{F} f)(g) \omega(g) U^{Z}(g) d g
$$

when the right hand side makes sense, is usually called the Weyl quantized of the function $f$ on $\hat{G}$, the continuous function $\omega$ from $G$ to $\mathbb{T}$ being such that

$$
\zeta^{\prime}\left(g, g^{\prime}\right)=\omega(g) \omega\left(g^{\prime}\right) \overline{\omega\left(g g^{\prime}\right)} \zeta\left(g, g^{\prime}\right)
$$

is a bicharacter.

In the general case, $\omega$ can be chosen quite arbitrarily and will be called ordering.

The quantized operator $Q(v)$ is just a multiplicative operator when $v$ takes some simple form. We generalize here a well known result for ordinary quantum mechanics :

Proposition 1.14. Using the assumptions and notations of Proposition 1.9 and Definition 1.11, if $v$ is a bounded measure on $G$ such that

$$
v=v_{0} \times \delta_{e},
$$


where $v_{0}$ is a bounded measure on $G_{0}$ and $\delta_{e}$ the evaluation in $e$ on $G_{1}$, then its quantized $Q(v)$ satisfies

$$
(Q(v) f)(k)=N(k) f(k), \quad k \in G_{1},
$$

the bounded continuous function $N$ being given by:

$$
N(k)=\int_{G_{0}} \lambda\left(g_{0}, e\right) \overline{\lambda(e, e)} \chi\left(g_{0}\right) b^{5}\left(\left(g_{0}, e\right),\left(e, k^{-1}\right)\right) d v_{0}\left(g_{0}\right) .
$$

\section{Perturbed Evolutions. Poisson's Formula}

As previously, let $G$ be a topological group, $\zeta$ be a multiplier on it. $G$ acts continuously on a topological space $\mathfrak{X}$, which has a positive quasi-invariant measure $\mu$ with respect to the action of $G$.

$U^{Z}$ is a unitary projective representation of $G$ on $L_{2}(\mathfrak{X}, \mu)$ associated to a function $Z$ which satisfies conditions of Proposition 1.4.

Let $t \rightarrow U_{t}^{0}$ be a unitary continuous one parameter group in $L_{\infty}(\mathfrak{X}, \mu)$ and let us define $H_{0}$ its infinitesimal generator

$$
\frac{d U_{t}^{0}}{d t}=-i H_{0} U_{t}^{0}
$$

Let us assume moreover that there exists a continuous function $h_{0}$ such that

$$
\left(H_{0} f\right)(x)=h_{0}(x) f(x)
$$

for a dense subset of vectors $f$ in $L_{2}(\mathfrak{X}, \mu)$.

Let $v$ be a bounded measure on $G$, and $Q(v)$ its quantized (see Definition 1.11).

Our aim in the following is to describe the solution of the equation

$$
\frac{d}{d t}\left(U_{t} f\right)=-i\left(H_{0}+Q(v)\right) U_{t} f,\left.\quad U_{t} f\right|_{t=0}=f
$$

for a dense set of functions $f$ in $L_{2}(\mathfrak{X}, \mu)$. The solution of such an equation for bounded $Q(v)$ can be obtained by the wellknown Dyson expansion. It is this expansion that we want to analyze in terms of Poisson process.

A generalized Poisson process on $G$ for the finite time interval $[0, T]$, corresponding to the bounded measure $m$ on $G$ is defined in the following way (see e.g. $[5,6])$.

$\Omega$ is the space

$$
\Omega=\left\{\omega=\left(n ; t_{1}, \ldots, t_{n}, g_{1}, \ldots, g_{n}\right) ; n \in \mathbb{N}, 0<t_{1}<t_{2} \ldots<t_{n}<T, g_{i} \in G\right\} \cup\left\{\omega_{0}\right\},
$$

where $\omega_{0}$ is a point. by

$\mathscr{F}$ is the Borel $\sigma$-algebra generated by the sets $\vartheta_{a_{1}, \ldots, a_{n} B_{1}, \ldots, B_{n}}^{(n)}$ which are defined

$$
\begin{aligned}
V^{(0)} & =\left\{\omega_{0}\right\} \\
V_{a_{1}, \ldots, a_{n} B_{1}, \ldots, B_{n}}^{(n)} & =\left\{\left(n, t_{1}, \ldots, t_{n}, g_{1}, \ldots, g_{n}\right)\right\},
\end{aligned}
$$

where the $B_{i}$ 's are sequences of Borel sets in $G$ and the $a_{i}$ 's are sequences of Borel sets of $[0, T]$ which satisfy $\forall t_{i} \in a_{i}, \forall t_{j} \in a_{j}, i<j \Rightarrow t_{i}<t_{j}$. 
Given $m$ a bounded measure on $G, P_{m}$ is the (bounded) measure on $\Omega$ such that

$$
\begin{aligned}
P_{m}\left(V^{(0)}\right) & =1 \\
P_{m}\left(V_{a_{1}, \ldots, a_{n} B_{1}, \ldots, B_{n}}^{(n)}\right) & =\prod_{i=1}^{n}\left|a_{i}\right| m\left(B_{i}\right),
\end{aligned}
$$

where $\left|a_{i}\right|$ is the Lebesgue measure of $a_{i}$.

If $F$ is a measurable function on $\Omega$ then its expectation value defined by

can be explicitly computed

$$
E^{T}(F)=\int_{\Omega} F(\omega) P(d \omega)
$$

$$
E^{T}(F)=\sum_{n=0}^{\infty} \int_{0}^{T} d t_{n} \int_{0}^{t_{n}} d t_{n-1} \ldots \int_{0}^{t_{2}} d t_{1} \int_{G} d m\left(g_{n}\right) \ldots \int_{G} d m\left(g_{1}\right) F\left(n, t_{i}, g_{i}\right) .
$$

Moreover a $G$ valued generalised Poisson process on $\Omega$ is defined in the following way:

$$
\begin{aligned}
& \left.\left.g(t)\left(n, t_{i}, g_{i}\right)=g_{k+1}^{-1} \ldots g_{n}^{-1}, \quad t \in\right] t_{k}, t_{k+1}\right], \quad k=1, \ldots, n-1 \\
& \left.\left.g(t)\left(n, t_{i}, g_{i}\right)=e, \quad t \in\right] t_{n}, T\right] \\
& g(t)\left(n, t_{i}, g_{i}\right)=g_{1}^{-1} \ldots g_{n}^{-1}, \quad t \in\left[0, t_{1}\right] .
\end{aligned}
$$

In fact we shall be interested by measurable functions $F$ defined as functionals on the previously introduced process. In order to underline this fact and noticing that every associated path ends at $g=e$, the identity of the group, we shall in the following denote the expectation value by $E_{g(T)=e}^{T}$. More precisely we shall be interested in the following measurable functions (or associated functionals):

Definition 2.8. Let $G$ be a topological group and $\zeta$ a continuous multiplier on $G$. $\Xi_{\zeta}(g)$ is the measurable functional defined on $\Omega$ by

$$
\begin{gathered}
\Xi_{\zeta}(g)\left(n, t_{i}, g_{i}\right)=\zeta\left(g\left(t_{n}\right)^{-1}, g\left(t_{n}\right) g\left(t_{n-1}\right)^{-1}\right) \\
\ldots \zeta\left(g\left(t_{k}\right)^{-1}, g\left(t_{k}\right) g\left(t_{k-1}\right)^{-1}\right) \ldots \zeta\left(g\left(t_{2}\right)^{-1}, g\left(t_{2}\right) g\left(t_{1}\right)^{-1}\right),
\end{gathered}
$$

where to simplify we have written:

$$
\begin{gathered}
g\left(t_{k}\right)=g\left(t_{k}\right)\left(n_{i}, t_{i} g_{i}\right) \\
\Xi_{\zeta}(g)\left(w_{0}\right)=\Xi_{\zeta}(g)\left(1, t_{1}, g_{1}\right)=1 .
\end{gathered}
$$

This functional has the obvious property:

Proposition 2.9. Let $\zeta$ and $\zeta^{\prime}$ be two equivalent multipliers, namely there exists a continuous function $\lambda$ from $G$ to the torus TI such that

$$
\zeta^{\prime}\left(g, g^{\prime}\right)=\lambda(g) \lambda\left(g^{\prime}\right) \overline{\lambda\left(g g^{\prime}\right)} \zeta\left(g, g^{\prime}\right) .
$$

Hence

and

$$
\begin{gathered}
\Xi_{\zeta}(g)\left(()_{0}\right)=\Xi_{\zeta}(g)\left(\omega_{0}\right) \\
\Xi_{\zeta}(g)\left(1, t_{1}, g_{1}\right)=\Xi_{\zeta}(g)\left(1, t_{1}, g_{1}\right)
\end{gathered}
$$

$$
\Xi_{\zeta}(g)\left(n, t_{i}, g_{i}\right)=\lambda\left(g_{1}\right) \ldots \lambda\left(g_{n}\right) \overline{\lambda\left(g_{n} \ldots g_{1}\right)} \Xi_{\zeta}(g)\left(n, t_{i}, g_{i}\right)
$$

Another measurable functional is important: 
Definition 2.10. If $h_{0}$ is a continuous function on $\mathfrak{X}$

$$
\omega \in \Omega \rightarrow \exp \left\{-i \int_{0}^{T} h_{0}\left(x g(\tau)^{-1}\right) d \tau\right\}(\omega)=\exp \left\{-i \sum_{k=1}^{n+1}\left(t_{k}-t_{k-1}\right) h_{0}\left(x g_{n+1} \ldots g_{k}\right)\right\}
$$

for $\omega=\left(n, t_{i}, g_{i}\right), t_{n+1}=T, g_{n+1} \ldots e$, defines a measurable functional on $\Omega$.

As a last example let us mention that if $f$ is any continuous function on $G$, the functional $\omega \in \Omega \rightarrow f\left(g(0)^{-1}\right)$ is measurable. Now we are able to state the main theorem.

Theorem 2.11. $G$ is a topological group and $\zeta$ a continuous multiplier on it. $G$ acts continuously on a topological space $\mathfrak{X}$ and $\mu$ is a positive quasi-invariant measure on $\mathfrak{X}$ with respect to the action of $G . U^{Z}$ is the unitary projective representation of $G$ on $L_{2}(\mathfrak{X}, d \mu)$ corresponding to the function $Z$ defined in Proposition 1.4.

$h_{0}$ is a continuous function on $\mathfrak{X}$ and $v$ a bounded measure on $G, \omega$ is an ordering factor and $H_{0}$ denotes the operator associated to $h_{0}$ on $L_{2}(\mathfrak{X}, d \mu)$. Denote by

$$
Q^{\omega}(v)=\int_{G} \omega(g) U^{Z}(g) d v(g)
$$

the quantized of $v$ with respect of the ordering $\omega$. Let $U_{s}$ be the solution (in $\mathfrak{M}$ ) of the equation

$$
\begin{aligned}
\frac{d}{d t} U_{s} & =-i\left(H_{0}+Q^{\omega}(v)\right) U_{s} \\
\left.U_{s}\right|_{s=0} & =1 .
\end{aligned}
$$

Then if $\dot{f}$ is a continuous function of $L_{2}(\mathfrak{X}, d \mu)$, for $x \in \mathfrak{X}$

$$
\left(U_{T} f\right)(x)=E_{g(T)=e}^{T}\left\{e^{-i \int_{0}^{T} h_{0}\left(x g(\tau)^{-1}\right) d \tau} \Xi_{\zeta}(g) Z\left(x, g(0)^{-1}\right) \sqrt{\frac{d \mu\left(x g(0)^{-1}\right)}{d \mu(x)}} f\left(x g(0)^{-1}\right)\right\},
$$

where $E_{g(T)=e}^{T}$ denotes the expectation with respect to the Poisson process on $G$ for the measure $d m(g)=-i \omega(g) d v(g)$.

Proof. The right-hand side is well defined according to the previous remarks. Moreover from (2.7)

$$
\begin{aligned}
& E_{g(T)=e}^{T}\left\{e^{-i \int_{0}^{T} h_{0}\left(x g(\tau)^{-1}\right) d \tau} \Xi_{\zeta}(g) Z\left(x g(0)^{-1}\right) \sqrt{\frac{d \mu\left(x g(0)^{-1}\right)}{d \mu(x)}} f\left(x g(0)^{-1}\right)\right\} \\
& =e^{-i T h_{0}(x)} f(x)+\sum_{n=1}^{\infty}(-i)^{n} \int_{0}^{T} d t_{n} \int_{0}^{t_{n}} d t_{n-1} \ldots \int_{0}^{t_{2}} d t_{1} \\
& \cdot \int_{G} \ldots \int_{G} \omega\left(g_{1}\right) \ldots \omega\left(g_{n}\right) d v\left(g_{1}\right) \ldots d v\left(g_{n}\right) \\
& \cdot e^{-i \sum_{k=1}^{n+1}\left(t_{k}-t_{k-1}\right) h_{0}\left(x g_{n+1} \ldots g_{k}\right)} \zeta\left(g_{n}, g_{n-1}\right) \zeta\left(g_{n} g_{n-1}, g_{n-2}\right) \\
& \ldots \zeta\left(g_{n} \ldots g_{2}, g_{1}\right) Z\left(x, g_{n} \ldots g_{1}\right) \sqrt{\frac{d \mu\left(x g_{n} \ldots g_{1}\right)}{d \mu(x)}} f\left(x g_{n} \ldots g_{1}\right) \text {, }
\end{aligned}
$$


where $g_{n+1}=e$ and $t_{n+1}=T$

$$
\begin{gathered}
=\left(e^{-i T H_{0}} f\right)(x)+\sum_{n=1}^{\infty}(-i)^{n} \int_{0}^{T} d t_{n} \int_{0}^{t_{n}} d t_{n-1} \ldots \int_{0}^{t_{2}} d t_{1} \int_{G} \ldots \int_{G} \\
\cdot \omega\left(g_{1}\right) \ldots \omega\left(g_{n}\right) d v\left(g_{1}\right) \ldots d v\left(g_{n}\right)\left\{e^{-i\left(T-t_{n}\right) H_{0}} \theta_{g_{n}}\left(e^{-i\left(t_{n}-t_{n-1}\right) H_{0}}\right)\right. \\
\cdot \theta_{g_{n} g_{n-1}}\left(e^{-i\left(t_{n-1}-t_{n-2}\right) H_{0}}\right) \ldots \theta_{g_{n} \ldots g_{k}}\left(e^{-i\left(t_{t_{n}}-t_{k-1}\right) H_{0}}\right) \\
\ldots \theta_{g_{n} \ldots g_{1}}\left(e^{-i t_{1} H_{0}}\right) \zeta\left(g_{n}, g_{n-1}\right) \zeta\left(g_{n} g_{n-1}, g_{n-2}\right) \\
\left.\ldots \zeta\left(g_{n} \ldots g_{2}, g_{1}\right) U^{Z}\left(g_{n} \ldots g_{1}\right) f\right\}(x) \\
=\left(e^{-i T H_{0}} f\right)(x)+\sum_{n=1}^{\infty}(-i)^{n} \int_{0}^{T} d t_{n} \int_{0}^{t_{n}} d t_{n-1} \ldots \int_{0}^{t_{2}} d t_{1} \\
\cdot \int_{G} \ldots \int_{G} \omega\left(g_{1}\right) \ldots \omega\left(g_{n}\right) d v\left(g_{1}\right) \ldots d v\left(g_{n}\right) \\
\left\{e^{-i\left(T-t_{n}\right) H_{0}} U^{Z}\left(g_{n}\right) e^{-i\left(t_{n}-t_{n-1}\right) H_{0}} U^{Z}\left(g_{n-1}\right) \ldots e^{-i\left(t_{2}-t_{1}\right) H_{0}} U^{Z}\left(g_{1}\right) e^{-i t_{1} H_{0}} f\right\}(x) \\
=\left(e^{-i T H_{0}} f\right)(x)+\sum_{n=1}^{\infty}(-i)^{n} \int_{0}^{T} d t_{n} \int_{0}^{t_{n}} d t_{n-1} \ldots \int_{0}^{t_{2}} d t_{1} \\
\left.\cdot e^{-i\left(T-t_{n}\right) H_{0}} Q^{\omega}(v) e^{-i\left(t_{n}-t_{n-1}\right) H_{0}} \ldots e^{-i\left(t_{2}-t_{1}\right) H_{0}} Q^{\omega}(v) e^{-i t_{1} H_{0}} f\right\}(x)
\end{gathered}
$$

which is precisely the Dyson expansion of $e^{-i\left(H_{0}+Q^{\omega}(v)\right) T}$.

In Theorem 2.11, the functional $\Xi_{\zeta}$ can be dropped in some important case:

Corollary 2.12. Under the same assumptions and notations as in Proposition 1.9 and Definition 1.11, assuming moreover that $v=\delta_{e} \times v_{1}$ where $v_{1}$ is a bounded measure on $G_{1}$, we have

$$
\begin{aligned}
\left\{U_{T} f\right\}(k)= & E_{g(T)=e}^{T}\left\{e^{-i_{\mathrm{O}}^{T} h_{0}\left(k g_{1}(\tau)^{-1}\right) d \tau} u(k) \overline{u\left(k g_{1}(0)^{-1}\right)}\right. \\
& \left.\cdot \sqrt{\frac{d \mu\left(k g_{1}(0)^{-1}\right)}{d \mu(k)}} f\left(k g_{1}(0)^{-1}\right)\right\}
\end{aligned}
$$

for u defined by

$$
u(k)=\lambda(e, k) v(k)
$$

and for the Poisson process on $G_{1}$ with measure

$$
d m\left(g_{1}\right)=-i \lambda\left(e, g_{1}\right) \omega\left(e, g_{1}\right) d v_{1}\left(g_{1}\right) .
$$

\section{Specific Examples in Generalized Quantum Mechanics}

In this section, we shall apply the previous results to specific examples, namely the usual quantum mechanics with $N$ degrees of freedom and also to spin systems in the Fock representation. All these results apply as well to more general situations where $G$ is of the form $\mathfrak{G} \times \hat{\mathfrak{G}}, \mathfrak{5}$ being an abelian locally compact group and the bicharacter $b^{\zeta}$ associated to the multiplier $\zeta$ being of the form:

$$
b^{\zeta}\left(\left(g_{1}, \hat{g}_{1}\right),\left(g_{2}, \hat{g}_{2}\right)\right)=\left(g_{2} \mid \hat{g}_{1}\right) \overline{\left(g_{1}, \hat{g}\right)},
$$

where $(\cdot \mid \cdot)$ denotes the duality between $\mathfrak{G}$ and $\hat{\mathfrak{G}},[8]$. 


\section{a) Quantum Mechanics with $N$ Degrees of Freedom}

In this case $\mathfrak{G}=\mathbb{R}_{N}$. $G=\mathbb{R}_{N} \times \mathbb{R}_{N}$ is an abelian locally compact group for the usual topology. We choose as multiplier:

$$
\zeta\left((x, p),\left(x^{\prime} p^{\prime}\right)\right)=\exp \left(-\frac{i}{2}\left(x p^{\prime}-x^{\prime} p\right)\right) .
$$

This multiplier restricted to $\mathbb{R}_{N} \times\{0\} \times \mathbb{R}_{N} \times \mathbb{R}_{N}$ (resp. $\{0\} \times \mathbb{R}_{N} \times \mathbb{R}_{N} \times \mathbb{R}_{N}$ ) is trivial

$$
\zeta\left((x, 0),\left(x^{\prime}, p^{\prime}\right)\right)=\exp \left(\frac{i}{2} x^{\prime} p^{\prime}\right) \exp \left(-\frac{i}{2}\left(x+x^{\prime}\right) p^{\prime}\right)
$$

hence

$$
\lambda(x, p)=\exp \left(\frac{i}{2} x p\right) \quad\left[\text { resp. } \exp \left(-\frac{i}{2} x p\right)\right] .
$$

The " $x$ " and " $p$ " representations can be written explicitly in $L_{2}\left(\mathbb{R}_{N}, d \xi\right)$ [resp. $\left.L_{2}\left(\mathbb{R}_{N}, d \pi\right)\right]$

$$
\left\{U^{x}(x, p) \psi\right\}(\xi)=\exp \left(-\frac{i}{2} x p-i \xi p\right) \psi(\xi+x)
$$

and

$$
\left\{U^{p}(x, p) \psi\right\}(\pi)=\exp \left(\frac{i}{2} x p+i \pi x\right) \psi(\pi+p)
$$

these two representations are intertwined by Fourier transformation.

Despite the complete symmetry between the two representations, the study of unitary groups generated by operators of the type $Q\left(h_{0}\right)+Q(v)$ singles out a representation since in general $h_{0}$ is just a continuous function on $\mathbb{R}_{N}$ which is not the Fourier transform of a bounded measure. Hence we can state the theorem.

Theorem 3.6. Let $h(x, p)=h_{0}(p)+v(x, p)$ be a classical hamiltonian on $\mathbb{R}_{N} \times \mathbb{R}_{N}$, where $h_{0}$ is a continuous function and $v(x, p)$ the symplectic Fourier transform of a bounded measure $v$ on $\mathbb{R}_{N} \times \mathbb{R}_{N}$ then:

$$
\begin{aligned}
\left\{e^{-i T Q^{1}(h)} \psi\right\}(\pi)= & E_{(x(T), p(T))=(0,0)}^{T} \\
& \cdot\left\{e^{-i \int_{0}^{T} h_{0}(\pi-p(\tau)) d \tau} e^{-i \int_{0}^{T} p(\tau) d x(\tau)-i \pi x(0)} \psi(\pi-p(0))\right\},
\end{aligned}
$$

where $E_{(x(T), p(T))=(0,0)}^{T}$ is the expectation of the Poisson process on $\mathbb{R}_{N} \times \mathbb{R}_{N}$ associated with the bounded measure $-i \exp \left(\frac{i}{2} x p\right) d v(x, p)$ and $Q^{1}(h)$ is defined in (1.13). Moreover the exponential $\exp \left(-i \int_{0}^{T} p(\tau) d x(\tau)\right)$ is the measurable function on $\Omega$ defined by:

$$
\exp \left(-i \int_{0}^{T} p(\tau) d x(\tau)\right)=\exp \left(-i \sum_{k=1}^{n} p\left(t_{k}\right)\left(x\left(t_{k}\right)-x\left(t_{k-1}\right)\right)\right.
$$

for $(x, p)(t)=(x, p)(t)(\omega), \omega \in \Omega$. 
This theorem is a direct application of Theorem 2.11 and Proposition 2.9. Let $\Xi_{\zeta}$ be the functional defined in (2.8)

$$
\begin{gathered}
\Xi_{\zeta}\left(\left(n, t_{1}, \ldots, t_{n},\left(x_{1}, p_{1}\right) \ldots\left(x_{n}, p_{n}\right)\right)\right. \\
\left.=\exp \left(-i \sum_{k=1}^{n} p\left(t_{k+1}\right)\left(x\left(t_{k+1}\right)-x\left(t_{k}\right)\right)\right)\right) \\
\cdot \prod_{k=1}^{n} \exp \left(\frac{i}{2} p_{k} x_{k}\right) \exp \left(-\frac{i}{2}\left(\sum_{k=1}^{n} p_{k}\right)\left(\sum_{k^{\prime}=1}^{n} x_{k^{\prime}}\right)\right)
\end{gathered}
$$

the factor $\prod_{k=1}^{n} \exp \left(\frac{i}{2} p_{k} x_{k}\right)$ can be embedded in the measure whereas the factor

$$
\exp \left(-\frac{i}{2}\left(\sum_{k} p_{k}\right)\left(\sum_{k^{\prime}} x_{k^{\prime}}\right)\right)
$$

cancels the same factor coming from the action of $U^{p}$.

The result is simpler for velocity independent potentials:

Proposition 3.7. Let $h_{0}$ be a continuous function on $\mathbb{R}_{N}$ and $V$ be the Fourier transform of a bounded measure $v$ on $\mathbb{R}_{N}$, let $f$ be a continuous function of $L_{2}\left(\mathbb{R}_{N}, d \pi\right)$

$$
\left.\left(e^{-i T\left(Q \left(h_{0}\right.\right.}+V\right) f\right)(\pi)=E_{p(T)=0}^{T}\left\{e^{-i \int_{0}^{T} h_{0}(\pi-p(\tau)) d \tau} f(\pi-p(0))\right\},
$$

where $E_{p(T)=0}^{T}$ is the expectation with respect to the Poisson process on $\mathbb{R}_{N}$ for the measure $-i d v$ and

$$
\left(h_{0}+V\right)(x, p)=h_{0}(p)+V(x) .
$$

Another example of the general theory is given by the gentle perturbations of classical evolution of quantum spin systems in the Fock representation.

\section{b) Quantum Spin Systems on a Lattice}

Let $\Lambda$ be an at most countable set, $\mathfrak{b}=\mathfrak{P}(\Lambda)$ the group of finite subsets of $\Lambda$ with respect to the symmetric difference denoted by $\triangle[9-11]$, its dual $\hat{\mathfrak{G}}=\mathscr{P}(\Lambda)$ the group of all subsets of $\Lambda$ for the same group law. Moreover choose

$$
\zeta\left((X, Y),\left(X^{\prime}, Y^{\prime}\right)\right)=(-1)^{\left|Y \cap X^{\prime}\right|}, \quad X, X^{\prime} \in \mathfrak{P}(\Lambda), \quad Y, Y^{\prime} \in \mathscr{P}(\Lambda),
$$

where $|X|$ denotes the cardinality of $X \in \mathfrak{P}(\Lambda)$. This example corresponds to spin systems in the Fock representation $[8,12]$. A classical Hamiltonian for a spin system is just a function $u_{0}: \mathfrak{P}(\Lambda) \rightarrow \mathbb{C}$ whose quantized $Q\left(u_{0}\right)$ acts on $l_{2}(\mathfrak{P}(\Lambda))$ as follows

$$
\left\{Q\left(u_{0}\right) \psi\right\}(X)=u_{0}(X) \psi(X) .
$$

We consider the potential $v: \mathscr{P}(\Lambda) \rightarrow \mathbb{C}$ which is continuous [hence bounded since $\mathscr{P}(\Lambda)$ is compact] and consider $\tilde{v}$ its Fourier transform which is a bounded measure on the discrete group $\mathfrak{P}(\Lambda)$. Then one has the following proposition.

Proposition 3.10. Let $u_{0}$ be a function from $\mathfrak{P}(\Lambda) \rightarrow \mathbb{C}$ and $v$ be a continuous function on $\mathscr{P}(\Lambda)$. Let $Q(v)$ the quantized of $v$ defined by:

$$
(Q(v) \varphi)(Y)=\sum_{\mathfrak{P}(\Lambda)} \tilde{v}(X) \varphi(X \triangle Y), \quad \varphi \in l_{2}(\mathfrak{P}(\Lambda))
$$


Then, for any $\psi \in l_{2}(\mathfrak{P}(\Lambda))$, we have:

$$
\left(e^{-i T\left(Q\left(u_{0}\right)+Q(v)\right)} \psi\right)(x)=E_{Y(T)=\emptyset}^{T}\left\{e^{-i \int_{0}^{T} u_{0}(X \triangle Y(\tau)) d \tau} \psi(X \triangle Y(0))\right\},
$$

where $E_{Y(T)=\emptyset}^{T}$ denotes the expectation with respect to the Poisson process whose sample paths are piecewise continuous functions with finite number of jumps in $\mathfrak{P}(\Lambda)$; the measure is $m(X)=-\tilde{v}(X)$.

In this paper we refrain to consider more general potentials.

A more exotic example is furnished by the group $\mathbb{Z} \times \mathbb{T}$ where $\mathbb{Z}$ is the additive group of natural numbers and $\mathbb{T}$ the torus. We shall not elaborate on it but postpone to a forthcoming paper where it is used to treat some bounded perturbation of the harmonic oscillator.

Before closing this section we want to make a remark: in the examples we considered, the groups are locally compact hence they possess an invariant measure, the Haar measure. This is not necessarily the case, for in Boson Field Theory (see next section) the group is the additive group of an infinite dimensional Hilbert space. Nevertheless, even for the case of locally compact groups, it can be of some interest to consider unitary projective representations corresponding to quasi-invariant measures. A well known example is given by the Bargmann representation [13] where the quasi-invariant measure is the Gaussian one.

\section{Specific Examples in Quantum Field Like Theories}

In this section we shall be interested by groups $G$ of the type

$$
G=\mathfrak{G}_{0} \times \mathfrak{b}_{1} .
$$

$\mathfrak{G}_{0}$ is a topological abelian group and $\mathfrak{5}_{1}$ is a subgroup of the dual of $\mathbf{6}_{0}$. $\zeta$ is a multiplier on $G$ such that the associated bicharacter $b^{\zeta}$ satisfies

$$
b^{\zeta}\left(\left(g_{0}, g_{1}\right),\left(g_{0}^{\prime}, g_{1}^{\prime}\right)\right)=\left(g_{0}^{\prime} \mid g_{1}\right) \overline{\left(g_{0} \mid g_{1}^{\prime}\right)} \quad g_{0}, g_{0}^{\prime} \in \mathfrak{G}_{0} \quad g_{1}, g_{1}^{\prime} \in \mathfrak{G}_{1}
$$

where $(\cdot \mid \cdot)$ denotes the duality between $\mathfrak{G}_{0}$ and $\hat{\mathfrak{G}}_{0}$. We shall treat three examples of such a situation.

a) $\mathbb{R}_{N} \times \mathbb{R}_{N}$ with Discrete Topology

We choose the same multiplier as previously

$$
\zeta\left((x, p),\left(x^{\prime}, p^{\prime}\right)\right)=\exp \left\{-\frac{i}{2}\left(x p^{\prime}-x^{\prime} p\right)\right\} .
$$

The dual group of $\mathbb{R}_{N}$, endowed with the discrete topology, is compact and strictly larger than $\mathbb{R}_{N}$. Beside the usual representation, there exists a lot of irreducible unitary projective representations of $\mathbb{R}_{N} \times \mathbb{R}_{N}$ which are not unitarily equivalent.

Let us consider for instance the "pure momentum" representation; in that case $\mathfrak{X}=\mathbb{R}_{N}$ taken with the discrete topology, the invariant measure being the counting measure. Hence the Hilbert space of the representation is $l_{2}\left(\mathbb{R}_{N}\right)$. The projective unitary representation of $\mathbb{R}_{N} \times \mathbb{R}_{N}$ on $l_{2}\left(\mathbb{R}_{N}\right)$ is formally the same as the usual " $p$ " 
representation, i.e.

$$
\{U(x, p) \psi\}(\pi)=\exp \left(\frac{i}{2} x p+i \pi x\right) \psi(\pi+p)
$$

except that now $\psi \in l_{2}\left(\mathbb{R}_{N}\right)$. More precisely, for every $\psi \in l_{2}\left(\mathbb{R}_{N}\right)$ there exists a countable set $\left\{\pi_{i}\right\}_{i \in \mathbb{Z}}, \pi_{i} \in \mathbb{R}_{N}$, such that

$$
\psi(\pi)=0 \quad \text { if } \quad \pi \notin\left\{\pi_{i}\right\}_{i \in \mathbb{Z}}
$$

and moreover

$$
\sum_{i \in \mathbb{Z}}\left|\psi\left(\pi_{i}\right)\right|^{2}<\infty
$$

Notice that the representation space is not separable. Nevertheless, within these representations, there exist pure momentum states

$$
\psi_{p_{0}}(\pi)= \begin{cases}1 & \text { if } \pi=p_{0} \\ 0 & \text { otherwise }\end{cases}
$$

For those states, one has

$$
\left\{U(x, p) \psi_{p_{0}}\right\}(\pi)=\exp \left\{-\frac{i}{2} x p+i x p_{0}\right\} \psi_{p_{0}-p}(\pi) .
$$

Consequently the $\psi_{p_{0}}$ are simultaneously eigenvectors of the $U(x, 0)$ with eigenvalue $\exp \left(i p_{0} x\right)$. As in the previous example, we can state:

Proposition 4.7. Let $G=\mathbb{R}_{N} \times \mathbb{R}_{N}$ be endowed with the discrete topology. Let $h_{0}$ be any function on $\mathbb{R}_{N}$ and $v$ the Fourier transform of a bounded measure on $\mathbb{R}_{N}$ (taken with discrete topology) and $\psi_{p_{0}}$ be a pure momentum state. Then

$$
\left\{e^{-i T\left(Q\left(h_{0}\right)+Q(v)\right)} \psi_{p_{0}}\right\}(\pi)=E_{\substack{p(T)=0 \\ p_{0}=\pi-p(0)}}^{T}\left\{e^{-i \int_{0}^{T} h_{0}(\pi-p(\tau)) d \tau}\right\} .
$$

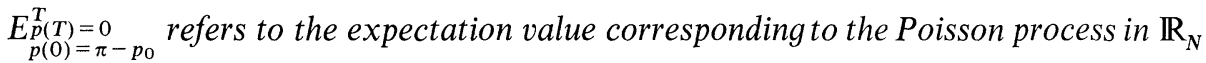
with jump measure - id $\tilde{v}$ and

$$
\left(h_{0}+v\right)(x, p)=h_{0}(p)+v(x) .
$$

Let us remark that in the case of velocity dependent potential, the previous expression can be extended:

$$
\begin{aligned}
& \left\{e^{-i T Q\left(h_{0}+v\right)} \psi_{p_{0}}\right\}(\pi) \\
& =E_{\substack{(x(T), p(T))=(0,0) \\
p(0)=\pi-p_{0}}}^{T}\left\{e^{-i \int_{0}^{T} h_{0}(\pi-p(\tau)) d \tau-i \int_{0}^{T} p(\tau) d x(\tau)-i \pi x(0)}\right\} .
\end{aligned}
$$

\section{b) Fermi Systems}

The example corresponding to Fermi systems is closely related to the previous example of spin systems [10]. Let us detail the formalism. $H$ is a real infinite dimensional separable Hilbert space with $s$ as fundamental real strictly positive symmetric bilinear form. A *-representation of the Clifford algebra $\overline{\mathfrak{I}(H, s)}[14]$ is given by a (real) linear map $x \in H \rightarrow b(x)$ into the (bounded) self-adjoint operators on a Hilbert space $\mathscr{H}$ which satisfies

$$
b(x)^{2}=s(x, x) \mathbb{1} .
$$


Moreover, let $\left\{e_{i}, f_{i}\right\}_{i \in \mathbb{Z}}$ be an orthonormal basis of $H$. Let $J$ be the complex structure on $H$ defined by

$$
J e_{i}=f_{i}, \quad J f_{i}=-e_{i}, \quad i \in \mathbb{Z} .
$$

Creation and annihilation operators associated with this complex structure are

$$
b_{J}^{ \pm}\left(\Gamma_{J} x\right)=\frac{1}{2}(b(x) \mp i b(J x)) \quad \forall x \in H,
$$

where $\Gamma_{J}$ is the identification map of $H$ to the complex Hilbert space whose scalar product is

$$
\left\langle\Gamma_{J}(x) \mid \Gamma_{J}(y)\right\rangle=s(x, y)+i s(J x, y) .
$$

Proposition 4.13. Let $\xi$ be the following multiplier on $\mathfrak{P}(\Lambda) \times \mathfrak{P}(\Lambda)$

$$
\xi\left((X, Y),\left(X^{\prime}, Y^{\prime}\right)\right)=\omega(X, Y) \omega\left(X^{\prime}, Y^{\prime}\right) \overline{\omega\left(X \triangle X^{\prime}, Y \triangle Y^{\prime}\right)}(-1)^{\left|Y \triangle \theta(X \triangle Y) \cap X^{\prime} \triangle \theta\left(X^{\prime} \triangle Y^{\prime}\right)\right|},
$$

where

$$
\omega(X, Y)=i^{|X \triangle \theta(X \triangle Y) \cap Y \triangle \theta(X \triangle Y)|}
$$

and $\theta$ is the unique homomorphism of $\mathfrak{P}(\Lambda)$ which satisfies

$$
\begin{aligned}
& \theta\left(\left\{x_{0}\right\}\right)=\emptyset \\
& \theta\left(\left\{x_{j}\right\}\right)=\left\{x_{i} \in \Lambda ; i<j\right\} \quad i>0
\end{aligned}
$$

for a given but arbitrary order on $\Lambda=\left\{x_{i}\right\}_{i \in \mathbb{Z}}$.

There exists a bijection between the unitary projective representations $U$ of $\mathfrak{P}(\Lambda) \times \mathfrak{P}(\Lambda)$ with multiplier $\xi$ and the $*$-representation of $\overline{\mathfrak{A}(H, s)}$ which is given by

$$
U(X, Y)=F(X, Y) \prod_{i \in X}^{<} b\left(e_{i}\right) \prod_{j \in Y}^{<} b\left(f_{j}\right),
$$

where $\prod^{<}$denotes the product in increasing order and $F$ is given by

$$
F(X, Y)=i^{|X \cap Y|+|\theta(X \triangle Y) \cap Y|+|X \cap \theta(X \triangle Y)|}(-1)^{|Y \cap \theta(Y)|+|X \triangle Y \cap \theta(X \triangle Y)|} .
$$

For the proof, see $[10$, p. 231] and [15].

As a special case

$$
\begin{gathered}
U\left(\left\{x_{i}\right\}, \emptyset\right)=b\left(e_{i}\right) \\
U\left(\emptyset,\left\{x_{i}\right\}\right)=b\left(f_{i}\right) \\
U\left(\left\{x_{i}\right\},\left\{x_{i}\right\}\right)=i b\left(e_{i}\right) b\left(f_{i}\right) .
\end{gathered}
$$

We can apply the general theory which has been developped to the group $\mathfrak{P}(\Lambda) \times \mathfrak{P}(\Lambda)$ with the multiplier $\xi$. However, both to simplify the proofs and to treat Fermi systems and quantum spin systems on lattice, we shall deal with the multiplier $\zeta$ defined in (3.8)

$$
\zeta\left((X, Y),\left(X^{\prime}, Y^{\prime}\right)\right)=(-1)^{\left|X^{\prime} \cap Y\right|} .
$$

This is possible since we have the following theorem [10]: 
Theorem 4.15. There is a bijection $\gamma$ between the projective unitary representation of $\mathfrak{P}(\Lambda) \times \mathfrak{P}(\Lambda)$ with multiplier $\xi$ and the projective unitary representation of $\mathfrak{P}(\Lambda) \times \mathfrak{P}(\Lambda)$ with multiplier $\zeta$

$$
\gamma\left(U^{\xi}(X, Y)\right)=\omega(X, Y) U^{\zeta}\left(\tau_{\theta}(X, Y)\right),
$$

where $\tau_{\theta}$ is an automorphism of $\mathfrak{P}(\Lambda) \times \mathfrak{P}(\Lambda)$ which satisfies

$$
\tau_{\theta}(X, Y)=(X \triangle \theta(X \triangle Y), Y \triangle \theta(X \triangle Y)) .
$$

Moreover $\tau_{\theta}$ leaves the diagonal $\Delta=\{(X, X) ; X \in \mathfrak{P}(\Lambda)\}$, invariant and satisfies $\tau_{\theta}^{2}=i$, the identity automorphism.

Hence we shall consider the group $\mathfrak{P}(\Lambda) \times \mathfrak{P}(\Lambda)$ with multiplier $\zeta \cdot \mathfrak{P}(\Lambda) \times \mathfrak{P}(\Lambda)$ acts on $\mathfrak{P}(\Lambda)$ according to

$$
Z \cdot(X, Y)=Z \triangle X \triangle Y
$$

and the counting measure on $\mathfrak{P}(\Lambda)$ is an invariant measure for this action. Moreover one has as the following lemma:

Lemma 4.16.

$$
\zeta\left((X, X),\left(X^{\prime}, Y^{\prime}\right)\right)=\lambda(X, X) \lambda\left(X^{\prime}, Y^{\prime}\right) \overline{\lambda\left(X \triangle X^{\prime}, Y \triangle Y^{\prime}\right)}
$$

with $\lambda(X, Y)=i^{|X|}$.

According to Proposition 1.9, there exists a unitary projective representation $U^{\zeta}$ of $\mathfrak{P}(\Lambda) \times \mathfrak{P}(\Lambda)$ on the space $l_{2}(\mathfrak{P}(\Lambda))$ which is given by

$$
\left(U^{\zeta}(X, Y) f\right)(Z)=i^{|X|}(-1)^{|X \cap Z|} f(Z \triangle X \triangle Y) \quad f \in l_{2}(\mathfrak{P}(\Lambda))
$$

this representation corresponds to Fock representation: as one can easily see the representation space contains a vacuum vector

$$
\Omega(Z)= \begin{cases}1 & \text { if } Z=\emptyset \\ 0 & \text { otherwise }\end{cases}
$$

Let $h_{0}$ be a real function on $\mathfrak{P}(\Lambda)$; it defines a self-adjoint operator on $l_{2}(\mathfrak{P}(\Lambda))$ by

$$
\left(H_{0} f\right)(Z)=h_{0}(Z) f(Z) \quad f \in l_{2}^{0}(\mathfrak{P}(\Lambda)),
$$

where $l_{2}^{0}(\mathfrak{P}(\Lambda))$ is the set of functions from $\mathfrak{P}(\Lambda) \rightarrow \mathbb{C}$ with finite support.

It is a matter of easy computation to see that the number operator

$$
N=\frac{1}{2} \sum_{i \in \mathbb{Z}}\left(1+i U^{\zeta}\left(\left\{x_{i}\right\},\left\{x_{i}\right\}\right)\right)
$$

and more generally an operator

$$
H_{0}=\sum_{i \in \mathbb{Z}} E_{i}\left(1+i U^{\zeta}\left(\left\{x_{i}\right\},\left\{x_{i}\right\}\right)\right) \quad E: \Lambda \rightarrow \mathbb{R}
$$

acts in that way with

$$
h_{0}(Z)=\sum_{i \in \mathbb{Z}} E_{i}\left\{1-(-1)^{\left|\left\{x_{i}\right\} \cap Z\right|}\right\} .
$$

Consequently according to Theorem 2.11 , we have: 
Proposition 4.20. Let $H_{0}$ be an operator in the Fock representation which can be written as

$$
H_{0}=\sum_{i \in \mathbb{Z}} E_{i}\left\{1+i U^{\zeta}\left(\left\{x_{i}\right\},\left\{x_{i}\right\}\right)\right\}
$$

Let $\mathbb{V}$ be a bounded operator of the form

$$
\mathbb{V}=\sum_{\mathfrak{P}(\Lambda) \times \mathfrak{P}(\Lambda)} V(X, Y) U^{\zeta}(X, Y),
$$

where $V$ is a bounded measure on $\mathfrak{P}(\Lambda) \times \mathfrak{P}(\Lambda)$. Then for any $f$ in $l_{2}(\mathfrak{P}(\Lambda)$ )

$$
\begin{gathered}
\left(e^{-i T\left(H_{0}+\mathbb{V}\right)} f\right)(Z)=i^{-|Z|} E_{(X(T), Y(T))=(\emptyset, \emptyset)}^{T} \\
\cdot\left\{e^{-i \int_{0}^{T} h_{0}(Z \Delta X(\tau) \Delta Y(\tau)) d \tau} e^{-i \pi \int_{0}^{T}|Y(\tau) \cap d X(\tau)|} i^{|X(0) \Delta Z|} f(Z \Delta X(0) \Delta Y(0))\right\},
\end{gathered}
$$

where as usual $E_{(X(T), Y(T))=(\emptyset, \emptyset)}^{T}$ denotes the expectation with respect to the Poisson process on $\mathfrak{P}(\Lambda) \times \mathfrak{P}(\Lambda)$ whose measure is $-i V(X, Y)$ and $\exp \left(-i \pi \int_{0}^{T}|Y(\tau) \cap d X(\tau)|\right)$ is a functional, on the sample paths set, defined by

$$
\begin{aligned}
& e^{-i \pi \int_{0}^{T}\left|Y(t)\left(n ; t_{i},\left(X_{i}, Y_{i}\right)\right) \cap d X(t)\left(n ; t_{i},\left(X_{i}, Y_{i}\right)\right)\right|} \\
& \quad=(-1)^{\sum_{k=1}^{n} \mid Y\left(t_{k+1}\right)\left(n ; t_{i} ;\left(X_{i} Y_{i}\right)\right) \cap\left(X\left(t_{k}+1\right)\left(n, t_{t}\left(X_{i} Y_{i}\right)\right) \cap X\left(t_{k}\right)\left(n, t_{t}\left(X_{i} Y_{i}\right)\right) \mid\right.}
\end{aligned}
$$

Corollary 4.21. Let $H_{0}$ be an operator in the Fock representation of canonical anticommutation relations which can be written as

$$
H_{0}=\sum_{i \in \mathbb{Z}} E_{i}\left\{1-U^{\xi}\left(\left\{x_{i}\right\},\left\{x_{i}\right\}\right)\right\} .
$$

Let $\mathbb{V}$ be a bounded operator of the form

$$
\mathbb{V}=\sum_{\mathfrak{P}(\Lambda) \times \mathfrak{P}(\Lambda)} V(X, Y) U^{\xi}(X, Y),
$$

where $V$ is a bounded measure on $\mathfrak{P}(\Lambda) \times \mathfrak{P}(\Lambda)$, then for any $f$ in $l_{2}(\mathfrak{P}(\Lambda))$

$$
\begin{gathered}
\left(e^{-i T\left(H_{0}+\mathbb{V}\right)} f\right)(Z)=i^{-|Z|} E_{X(T)=Y(T)=\emptyset}^{T} \\
\cdot\left\{e^{-i \int_{0}^{T} h_{0}(Z \Delta X(\tau) \triangle Y(\tau)) d \tau} e^{-i \pi \int_{0}^{T}|Y(\tau) \cap d X(\tau)|} i^{|X(0) \triangle Z|} f(Z \triangle X(0) \triangle Y(0))\right\},
\end{gathered}
$$

where $E_{X(T)=Y(T)=\emptyset}^{T}$ is the expectation with respect to the Poisson process on $\mathfrak{P}(\Lambda) \times \mathfrak{P}(\Lambda)$ whose bounded measure is $i^{(|X \cap Y|-1)} V\left(\tau_{\theta}(X, Y)\right)$, and $\tau_{\theta}$ as being previously defined.

\section{c) A Model of Bose Field Theory}

This last example is devoted to a very simple model of field theory to show that even in that case the previous considerations can apply. Let $G=H \times H$, where $H$ is an infinite dimensional real vector space, with the discrete topology. To be specific, let us consider the more special case where $H=\mathscr{D}_{R}\left(\mathbb{R}_{3}\right)$ is the vector space of real functions of $\mathbb{R}_{3}$ indefinitely differentiable with compact support. 
The symplectic form on $H \times H$ is given by

$$
\sigma\left((f, g),\left(f, g^{\prime}\right)\right)=\frac{1}{2} \int_{\mathbb{R}_{3}}\left(f^{\prime}(p) g(p)-f(p) g^{\prime}(p)\right) d p
$$

which is clearly non degenerate.

The multiplier one chooses is

$$
\zeta\left((f, g),\left(f^{\prime}, g^{\prime}\right)\right)=\exp \left(i \sigma\left((f, g),\left(f^{\prime}, g^{\prime}\right)\right)\right.
$$

this multiplier restricted to the subgroup $H \times\{0\} \times G$ is trivial.

On $H$ there exists an invariant measure, namely the counting measure. That measure would lead to a Hilbert space $l_{2}(H)$ which is non separable. Nevertheless there exists on $H$ quasi-invariant measures.

Let us consider a norm on $H$, for example

$$
\|f\|^{2}=\int_{\mathbb{R}_{3}} f(p)^{2} d p .
$$

The Gaussian measure $\mu$ associated with that norm is quasi-invariant, namely

$$
\sqrt{\frac{d \mu(h+g)}{d \mu(h)}}=\exp \left(-\int_{\mathbb{R}_{3}} g(p) h(p)-\frac{1}{2}\|g\|^{2}\right) .
$$

Let $\bar{H}$ be the Hilbert space closure of $\mathscr{D}_{R}\left(\mathbb{R}_{3}\right)$ with respect to this norm. $\mathscr{H}^{(0)}$ is the vector space of functions from $\bar{H}$ to $\mathbb{C}$ which depend only on a finite vector subspace of $\bar{H}$ (cylindrical functional) and which are square integrable with respect to the Gaussian measure. $\mathscr{H}$ is the completion of $\mathscr{H}^{(0)}$.

Corresponding to the Gaussian measure, one has, by Proposition 1.9, a representation of $H \times H$ on $\mathscr{H}$ which is given by

$$
\begin{gathered}
\{U(f, g) \Psi\}(h) \\
=\exp \left(\frac{i}{2} \int_{\mathbb{R}_{3}} f(p) g(p) d p+i \int_{\mathbb{R}_{3}} f(p) h(p) d p-\int_{\mathbb{R}_{3}} g(p) h(p) d p-\frac{1}{2}\|g\|^{2}\right) \Psi(h+g)
\end{gathered}
$$

$\Psi \in \mathscr{H}$. Notice that $U$ leaves $\mathscr{H}^{(0)}$ invariant. The unitary groups $\alpha \in \mathbb{R} \rightarrow U(\alpha f, 0)$ and $\beta \in \mathbb{R} \rightarrow U(0, \beta g)$ are continuous. Their infinitesimal generators are respectively

$$
\{\Pi(f) \Psi\}(h)=\int_{\mathbb{R}_{3}} f(p) h(p) d p \Psi(h)
$$

and

$$
\{\Phi(g) \Psi\}(h)=i \int_{\mathbb{R}_{3}} g(p) h(p) d p \Psi(h)-i\left(\frac{\delta \Psi}{\delta g}\right)(h)
$$

for $\Psi$ such that

$$
\left(\frac{\delta \Psi}{\delta g}\right)(h)=\lim _{\lambda \rightarrow 0} \frac{1}{\lambda}\{\Psi(h+\lambda g)-\Psi(h)\}
$$

exists.

Let us consider the functional $\Omega \in \mathscr{H}^{(0)}$

$$
\Omega(h)=1, \quad \forall h \in \bar{H}
$$

then

$$
\{\pi(g)+i \phi(g)\} \Omega=0
$$


which shows that the cyclic component of $\Omega$ with respect to $U$ is nothing but the usual Fock representation.

Consequently, one can apply the previous results to $H_{0}$ of the form

$$
H_{0}=P\left(\pi\left(g_{1}\right) \ldots \pi\left(g_{n}\right)\right) \text {, }
$$

where $P$ is a polynomial in $n$ variables, and even to

$$
H_{0}=\sum_{i \in \mathbb{Z}} \pi\left(g_{i}\right)^{2}
$$

where $f_{i}$ (resp. $g_{i}$ ) is an orthonormal basis in $\bar{H}$. The potential may be taken of the form

$$
\mathbb{V}=\sum_{i, j \in \mathbb{Z}} V_{i j} U\left(f_{i}, g_{j}\right) \quad \text { with } \quad \sum_{i, j \in \mathbb{Z}}\left|V_{i j}\right|<\infty
$$

Thus we can write a Feynman formula for that model:

$$
\begin{gathered}
\left(e^{-i T\left(H_{0}+\mathbb{V}\right)} \Psi\right)(h)=E_{f(T)=g(T)=0}^{T} \\
\cdot\left\{e^{-i \int_{0}^{T} h_{0}(h-g(\tau)) d \tau} e^{-i \int_{0}^{T}\langle g(\tau) \mid d f(\tau)\rangle} e^{-i\langle h \mid f(0)+i g(0)\rangle-1 / 2\|g(0)\|^{2}} \Psi(h-g(0))\right\},
\end{gathered}
$$

where the Poisson process is taken in $H \times H$ for the discrete measure which is defined in the following way:

$$
-i V(f, g)=\left\{\begin{array}{l}
-i \exp \left(\frac{i}{2}\left\langle f_{i} \mid g_{j}\right\rangle\right) V_{i j} \quad \text { if } f=f_{i}, \quad g=g_{j} \\
0 \text { otherwise, }
\end{array}\right.
$$

where the brackets denote the scalar product in $\bar{H}$.

\section{Appendix}

Theorem. Let $G$ be a topological Abelian group and $H$ a closed (invariant) subgroup of $G$. Let $g \in G \rightarrow[g] \in G / H$ be the canonical surjective homomorphism of $G$ onto $G / H$. Let $\zeta$ be a continuous multiplier on $G$ such that there exists a continuous function $\lambda: G \rightarrow T$ with the property

$$
\zeta(h, g)=\lambda(h) \lambda(g) \overline{\lambda(h g)}, \quad \forall h \in H, \quad \forall g \in G .
$$

Let us consider the natural action of $G$ onto $H / G$

$$
\left[g^{\prime}\right] g=\left[g^{\prime} g\right], \quad\left[g^{\prime}\right] \in G / H, \quad g \in G
$$

and let $\mu$ be a positive quasi-invariant measure on $G / H$ for this action. Then any unitary projective representation $U$ of $G$ onto $L_{2}(G / H, d \mu)$ is given by

$$
(U(g) f)\left(\left[g^{\prime}\right]\right)=\overline{\lambda\left(g^{\prime \prime}\right)} \lambda\left(g^{\prime \prime} g\right) \zeta\left(g^{\prime \prime}, g\right) \chi_{0}(g) u\left(\left[g^{\prime}\right]\right) \overline{u\left(\left[g^{\prime} g\right]\right)} \sqrt{\frac{d \mu\left[g^{\prime} g\right]}{d \mu\left[g^{\prime}\right]}} f\left(\left[g^{\prime} g\right]\right)
$$

for some character $\chi_{0}$ of $G$, some continuous function $u$ from $G / H$ to $T$ and for $g^{\prime \prime}$ arbitrary element of $G$ such that

$$
\left[g^{\prime \prime}\right]=\left[g^{\prime}\right]
$$


Conversely for $u$ and $\chi_{0}$ the previously formula defines an unitary projective representation of $G$.

Proof. Let $Z: G / H \times G \rightarrow T$ be defined by

$$
\left.\left(U^{Z}(g)\right) f\right)\left(\left[g^{\prime}\right]\right)=Z\left(\left[g^{\prime}\right], g\right) \sqrt{\frac{d \mu\left[g^{\prime} g\right]}{d \mu\left[g^{\prime}\right]}} f\left(\left[g^{\prime} g\right]\right) .
$$

Of course $Z$ satisfies $(1,4)$

$$
Z\left(\left[g^{\prime}\right], g_{1}\right) Z\left(\left[g^{\prime} g_{1}\right], g_{2}\right)=\zeta\left(g_{1}, g_{2}\right) Z\left(\left[g^{\prime}\right], g_{1} g_{2}\right) \text {. }
$$

Defining $U^{Z^{\prime}}(g)=\overline{\lambda(g)} U^{Z}(g)$ allows to consider only the case where $\zeta(h, g)=1$, $\forall h \in H$ and $g \in G$. In such a way, one has

$$
\zeta\left(h g, g^{\prime}\right)=\zeta\left(g, g^{\prime}\right) \text {. }
$$

Now let us define

$$
Z\left(\left[g^{\prime}\right], g\right)=\zeta\left(g^{\prime \prime}, g\right) w\left(\left[g^{\prime}\right], g\right),
$$

where $g^{\prime \prime}$ is any element in $G$ such that $\left[g^{\prime \prime}\right]=\left[g^{\prime}\right]$. Hence $w$ satisfies

$$
w\left(\left[g^{\prime}\right], g\right) w\left(\left[g^{\prime} g_{1}\right], g_{2}\right)=w\left(\left[g^{\prime}\right], g_{1} g_{2}\right) .
$$

From this equation, we see that the restriction of $w$ to $H$ is a character of $H$

$$
w\left(\left[g^{\prime}\right], h_{1} h_{2}\right)=w\left(\left[g^{\prime}\right], h_{1}\right) w\left(\left[g^{\prime}\right], h_{2}\right) \quad \forall h_{1}, h_{2} \in H .
$$

Take

$$
\chi_{\left[g^{\prime}\right]}(h)=w\left(\left[g^{\prime}\right], h\right)
$$

Let us now compute in two ways

$$
\begin{aligned}
(U(g) U(h) f)\left(g^{\prime}\right) & =Z\left(\left[g^{\prime}\right], g\right) \zeta\left(g^{\prime \prime} g, h\right) \chi_{\left[g^{\prime} g\right]}(h) f\left(\left[g^{\prime} g\right]\right) \\
& =b_{\zeta}(g, h) \zeta\left(g^{\prime \prime} h\right) \chi_{\left[g^{\prime}\right]}(h) Z\left(\left[g^{\prime}\right], g\right) f\left(\left[g^{\prime} g\right]\right) .
\end{aligned}
$$

Hence

$$
\zeta\left(g^{\prime \prime} g, h\right) \chi_{\left[g^{\prime} g\right]}(h)=b_{\zeta}(g, h) \zeta\left(g^{\prime \prime}, h\right) \chi_{\left[g^{\prime}\right]}(h) .
$$

With the condition $\zeta(h, g)=1$ we have

$$
\chi_{\left[g^{\prime} g\right]}(h)=\chi_{\left[g^{\prime}\right]}(h), \quad \forall h \in H, \quad g \in G
$$

so $\chi_{\left[g^{\prime}\right]}$ does not depend on the point in $G / H$ and defines a fixed character $\chi_{0}$ of $H$.

Given $\chi_{0}$ a character of $H$, there exists $\chi_{0}^{c}$ character of $G$ which extends $\chi_{0}$.

Hence we can write

$$
w\left(\left[g^{\prime}\right], g\right)=\chi_{0}^{c}(g) w^{\prime}\left(\left[g^{\prime}\right], g\right),
$$

where $w^{\prime}$ becomes 1 on $H$.

Come back to $(*)$, we can write:

$$
w\left(\left[g^{\prime}\right], g\right)=w\left(\left[g^{\prime}\right], g_{1} g_{2}\right) \overline{w\left(\left[g^{\prime} g_{1}\right], g_{2}\right)} .
$$


Taking $g_{2}=g_{1}^{-1} g^{\prime \prime-1}$ with $\left[g^{\prime \prime}\right]=\left[g^{\prime}\right]$

and

$$
w\left(\left[g^{\prime}\right], g_{1}\right)=w\left(\left[g^{\prime}\right], g^{\prime \prime-1}\right) \overline{w\left(\left[g^{\prime} g_{1}\right],\left(g^{\prime \prime} g_{1}\right)^{-1}\right)}
$$

$$
w^{\prime}\left(\left[g^{\prime}\right], g_{1}\right)=w^{\prime}\left(\left[g^{\prime}\right], g^{\prime \prime-1}\right) \overline{w^{\prime}\left(\left[g^{\prime} g_{1}\right],\left(g^{\prime \prime} g_{1}\right)^{-1}\right)}
$$

with $w^{\prime}\left(\left[g^{\prime}\right], h\right)=1 \quad \forall h \in H$.

Hence $w^{\prime}\left(\left[g^{\prime}\right], g^{\prime \prime-1}\right)$ only depends on the class $\left[g^{\prime}\right]$; we take

$$
u\left(\left[g^{\prime}\right]\right)=w^{\prime}\left(\left[g^{\prime}\right], g^{\prime \prime-1}\right) \quad\left[g^{\prime \prime}\right]=\left[g^{\prime}\right]
$$

then

$$
w\left(\left[g^{\prime}\right], g_{1}\right)=\chi_{0}^{c}\left(g_{1}\right) u\left(\left[g^{\prime}\right] \overline{u\left(\left[g^{\prime} g_{1}\right]\right)}\right.
$$

and

$$
\left(U^{Z^{\prime}}(g) f\right)\left(\left[g^{\prime}\right]\right)=\zeta\left(g^{\prime \prime}, g\right) \chi_{0}^{c}(g) u\left(\left[g^{\prime}\right]\right) \overline{u\left(\left[g^{\prime} g\right]\right)} \sqrt{\frac{d \mu\left[g^{\prime} g\right]}{d \mu\left[g^{\prime}\right]}} f\left(\left[g^{\prime} g\right]\right)
$$

Moreover

$$
\begin{aligned}
\left(U^{2}(g) f\right)\left(\left[g^{\prime}\right]\right)= & \overline{\lambda\left(g^{\prime \prime}\right)} \lambda\left(g^{\prime \prime} g\right) \zeta\left(g^{\prime \prime}, g\right) \chi_{0}^{c}(g) u\left(\left[g^{\prime}\right]\right) \overline{u\left(\left[g^{\prime} g\right]\right)} \\
& \cdot \sqrt{\frac{d \mu\left[g^{\prime} g\right]}{d \mu\left[g^{\prime}\right]}} f\left(\left[g^{\prime} g\right]\right),
\end{aligned}
$$

where $\zeta(h, g)=\lambda(h) \lambda(g) \overline{\lambda(h g)}$ and $\left[g^{\prime \prime}\right]=\left[g^{\prime}\right]$.

\section{References}

1. Feynman, R.P.: Rev. Mod. Phys. 20, 367 (1948)

2. Albeverio, S., Høegh-Krohn, R.: Mathematical theory of Feynamn-Path integrals. Lecture notes in mathematics, Vol. 523. Berlin, Heidelberg, New York: Springer 1976

3. Dewitt-Morette, C., Maheshwari, A., Nelson, B.: Phys. Rep. 50, 255-372 (1979)

4. Nelson, E.: J. Math. Phys. 5, 332 (1964)

5. Maslov, V.P., Chebotarev, A.M.: Soviet Math. Dok. 17, 4, 975 (1976)

6. Chebotarev, A.M., Maslov, V.P.: Processus de sauts et leurs applications dans la mécanique quantique. Proceedings of the conference on Feynman-Path integrals, Marseille 1978. In: Lecture notes in physics, Vol. 106. Berlin, Heidelberg, New York: Springer 1979

7. Mackey, G.W.: Functional analysis and related fields (ed.) Browder, F.E. Berlin, Heidelberg, New York: Springer 1970

8. Mackey, G.W.: Acta Math. 99, 265 (1958)

9. Chevalley, C.: Lie groups. Princeton, N.Y.: Princeton University Press 1946

10. Combe, Ph., Rodriguez, R., Sirugue-Collin, M., Sirugue, M.: Commun. Math. Phys. 63, 219 (1978)

11. Combe, Ph., Rodriguez, R., Sirugue-Collin, M., Sirugue, M.: J. Math. Phys. 20, 611 (1979)

12. Simon, B.: Mathematics of contemporary physics. Streater, R.F. (ed.). London, New York: Academic Press 1972

13. Bargmann, V.: Communications on pure and applied mathematics 14, 187 (1961)

14. Balslev, E., Manuceau, J., Verbeure, A.: Commun. Math. Phys. 8, 315 (1968)

15. Rodriguez, R.: Thèse de doctorat d'état, Marseille (1978)

Communicated by H. Araki

Received December 17, 1979 